\title{
Decreasing Number of Urodynamics in Urological and Gynaecological Clinics Reflects Decreased Importance for Surgical Indications: German Population-Based Data from 2013 to 2019
}

\author{
Martin Baunacke ${ }^{a} \quad$ Isabel Leuchtweis ${ }^{a} \quad$ Albert Kaufmann $^{b} \quad$ Marcel Schmidt $^{c}$ \\ Christer Groeben $^{a} \quad$ Angelika Borkowetz $^{a} \quad$ Nicole Eisenmenger $^{d}$ \\ Christian Thomas $^{a}$ Johannes Huber ${ }^{a}$

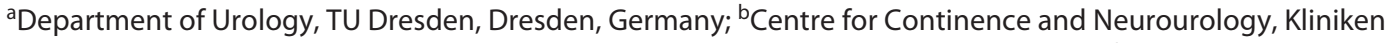

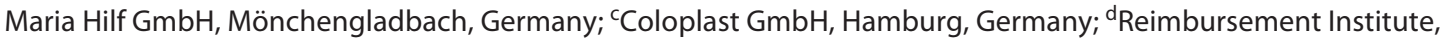 \\ Hürth, Germany
}

\section{Keywords \\ Urodynamics · Epidemiology · Health services research . Lower urinary tract symptoms . Urinary incontinence}

\begin{abstract}
Introduction: The routine use of urodynamic studies (UDS) has been questioned. Additionally, the material and personnel costs are poorly remunerated. We aimed to analyse the UDS utilization in Germany. Methods: We analysed UDS performed by hospitals based on quality reports from 2013 to 2019. A representative sample of 4 million insured persons was used to estimate outpatient UDS utilization from 2013 to 2018. Results: There was an overall decrease of $14 \%$ in UDS in Germany from 2013 to 2018 (60,980 to 52,319; $p=$ $0.003)$. In the outpatient sector, there was a slight non-continuous drop of $11 \%$ from 34,551 to 30,652 from 2013 to $2018(p=0.06)$. UDS utilization in hospitals decreased by $26 \%$ from 26,429 in 2013 to 19,453 in 2019 ( $p=0.004)$. University hospitals showed a smaller decrease $(3,007$ to 2,685 ; $p=0.02)$. In urology, the number of UDS $(11,758$ to 6,409 ; $p<0.001$ ) and the number of performing departments (328
\end{abstract}

karger@karger.com www.karger.com/uin

Karger $\stackrel{\text { ! }}{\div}$
C 2021 The Author(s).

Published by S. Karger AG, Basel

This is an Open Access article licensed under the Creative Commons Attribution-NonCommercial-4.0 International License (CC BY-NC) (http://www.karger.com/Services/OpenAccessLicense), applicable to the online version of the article only. Usage and distribution for commercial purposes requires written permission. to 263 clinics; $p<0.001)$ decreased. Gynaecological departments also showed a decrease in UDS $(1,861$ to $866 ; p<$ 0.001 ) and performing departments (159 to $68 ; p<0.001$ ). However, in paediatrics, there was an increase in UDS $(1,564$ to 2,$192 ; p=0.02$ ). By age, the number of children remained constant $(1,371$ to 1,$252 ; p=0.2)$, but there was a strong decrease seen in 60 - to 79 -year-olds $(9,792$ to 5,$564 ; p<0.001)$. Conclusion: UDS appear to be less important in the indication for surgery. Despite high resource expenditure and low remuneration, the decrease in urodynamics in the outpatient sector is less pronounced, indicating a trend to perform UDS in an outpatient setting.

(c) 2021 The Author(s).

Published by S. Karger AG, Basel

\section{Introduction}

Urodynamic studies (UDS) provide a direct assessment of lower urinary tract function by measuring relevant physiological parameters. UDS can play a critical role in the diagnosis of urinary incontinence, urge symptoms, neurogenic bladder, or lower urinary tract symp- 
toms (LUTS) [1,2]. While the diagnostic value of UDS is very high, there are some pitfalls that prevent the routine use of UDS. A good-quality technique is needed to obtain reliable results, preferably via standardized procedures like those provided by the International Continence Society (ICS) $[1,3,4]$. The testing can be uncomfortable for patients, and it carries a small risk of urinary tract infections [5]. Furthermore, the required resources of personnel, time, and material costs are often confronted with a low remuneration [6]. Therefore, several studies have been performed in the last 10 years to question the indication of UDS in specific clinical situations. The 2012 ValUE (Value of Urodynamic Evaluation) trial examined the influence of UDS on the outcome of women with uncomplicated stress urinary incontinence (SUI) [7]. It showed that office evaluation without UDS was not inferior to evaluation with UDS for outcomes at 1 year. In addition, the VUSIS 2 (the Value of Urodynamics before Stress Incontinence Surgery) trial showed no inferiority after midurethral sling operations of women with uncomplicated SUI who did not undergo UDS [8]. As a result, there were changes made in 2013 to the EAU (European Association of Urology) guidelines for urinary incontinence. The recommendation to "perform UDS prior to surgery for urinary incontinence if there are either symptoms of overactive bladder, a history of previous surgery, or a suspicion of voiding difficulty" was removed [9]. The routine use of UDS in men with LUTS has also been questioned [10]. The recent UPSTREAM Trial (Urodynamics for Prostate Surgery Trial: Randomized Evaluation of Assessment Methods) showed no benefit of UDS for surgery rates or outcomes during the routine diagnosis of men with LUTS [11].

Because of these studies and changes to the guidelines, there might have been a decline in UDS in recent years. However, there are no studies to date that have examined the trends of UDS utilization in recent years. The aim of this study was to analyse the use of UDS in Germany over time.

\section{Materials and Methods}

For this study, we analysed 2 data sets. To analyse the utilization of UDS by hospitals in Germany, we used German hospitals' quality reports. Since 2005, German hospitals have been legally obligated to provide information about their work and structures in quality reports. The quality reports include procedures that were performed as in- and outpatients by the hospitals. Data by age and sex are available from DESTATIS (Statistisches Bundesamt) and included only the inpatient cases. UDS were defined by OPS (Operationen-und Prozedurenschlüssel) code 1-334, including codes

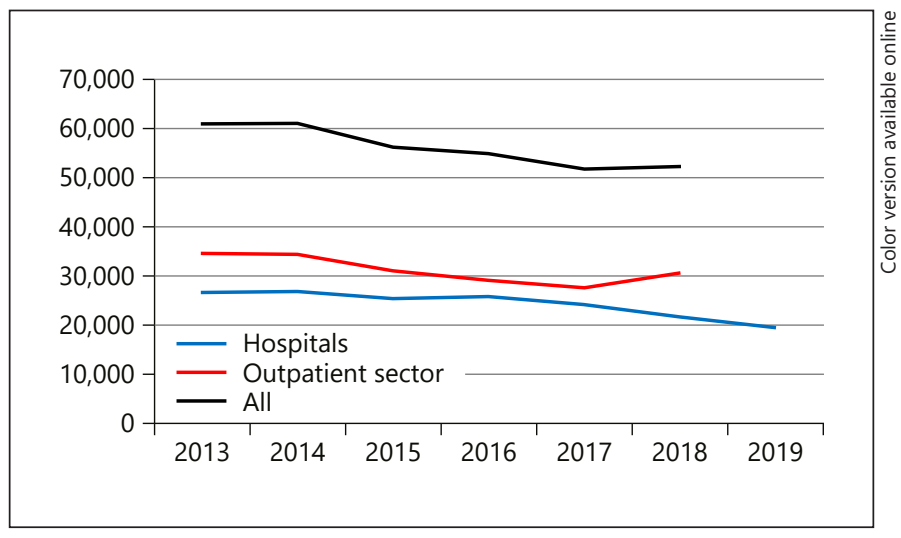

Fig. 1. UDS utilization in hospitals (2013-2019) and the outpatient sector (2013-2018).

1-334.0, 1-334.1, 1-334.2, 1-334.x, and 1-334.y. Departments were classified by the department code (FAB - Fachabteilungsschlüssel: urology 2200, gynaecology 2400, and paediatrics 1000). Paediatrics included paediatric surgery. Multidisciplinary centres are centres including several disciplines. Most of these centres are centres for patients with spinal cord injuries/paraplegics or are multidisciplinary neurourological centres, coded with 3700 (Miscellaneous), 2300 (Orthopaedics), or 2800 (Neurology). In the quality reports, 1-3 UDS per year are anonymized and displayed as a case number of 3 .

The quality of the hospitals' quality reports depends on the documentation of each hospital. We checked and corrected quality report data regarding errors in the documentation of the correct department code (FAB). Due to a high discrepancy, there was one correction of UDS case numbers in consultation with the responsible centre (A.K.). We used the analysis tool "reimbursement. INFO" (RI Innovation GmbH, Hürth, Germany) to extract data on hospital UDS utilization for the years 2013-2019. Hospitals and departments were classified into university and non-university hospitals according to the quality reports. For the analysis of UDS utilization in certified pelvic floor centres, we analysed only centres that were continuously certified by the German Continence Society (Deutsche Kontinenz Gesellschaft e.V.) from 2013 to 2018. Due to a restructuring of the certification process, no certifications took place in 2019. However, we analysed the UDS case numbers of these centres from 2013 to 2019.

To analyse the utilization of UDS by the outpatient sector, we used health insurance data from the InGef (Institute for Applied Health Research, Berlin, Germany) Research Database. The InGef Research Database comprises longitudinal data from approximately 6.7 million persons insured in one of the 70 German statutory health insurance plans. For this analysis, we used a sample of approximately 4 million insured people, which is representative of the German population in terms of age and sex. This sample has high external validity regarding overall measures of morbidity, mortality, and drug use [12]. Outpatient procedures were coded according to the EBM (Einheitlicher Bewertungsmaßstab). For UDS utilization, we analysed EBM code 26313 for the years 20132018. Due to the nature of data (health insurance data and public accessible hospital quality reports), an ethical approval was not 


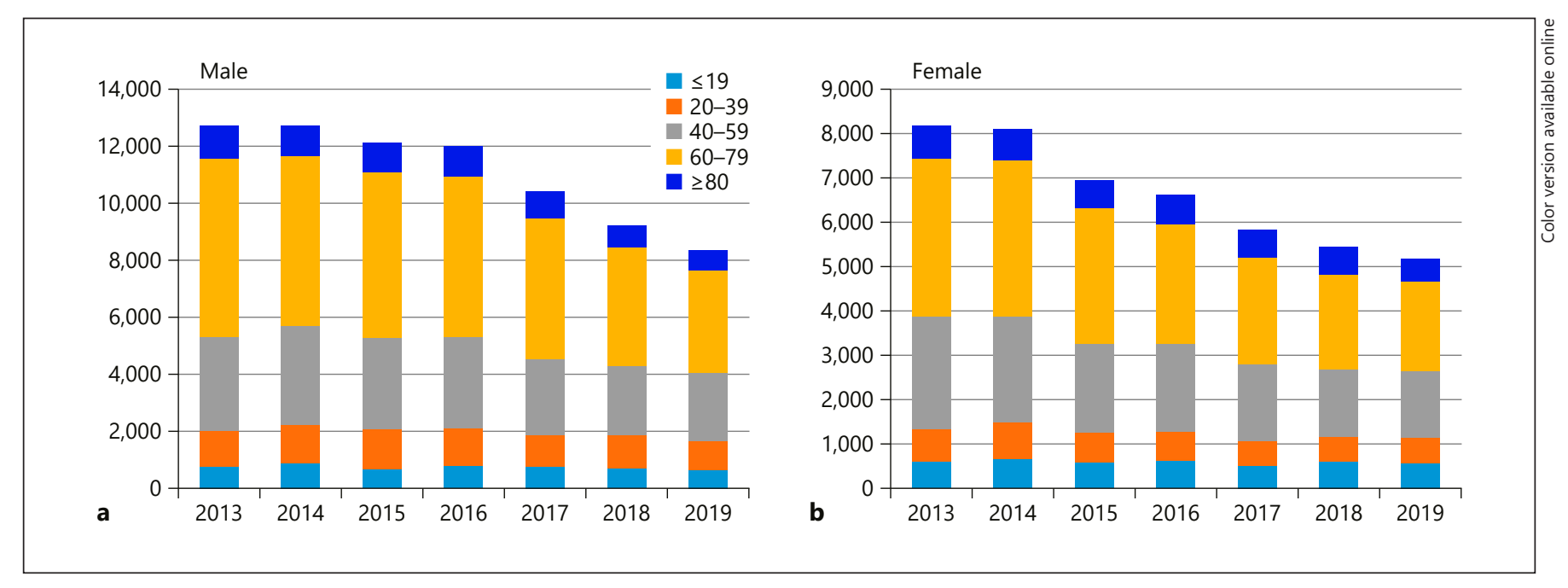

Fig. 2. a, b UDS utilization depending on age and gender from 2013 to 2019.

required. We applied linear regression analyses to analyse the trends over time. $p<0.05$ was considered to indicate significance. All calculations were performed with "IBM SPSS Statistics 27" (Armonk, NY, USA).

\section{Results}

There was an overall decrease of $14 \%$ in UDS utilization in Germany from 2013 to 2018 (60,980 to 52,319; $-2,073 \pm 328 \mathrm{UDS} /$ year; $p=0.003)$. In the outpatient sector, there was a slight non-continuous decrease of $-11 \%$ from 34,551 to 30,652 from 2013 to 2018 (-1,185 \pm 445; $p=0.06)$. In German hospitals, UDS numbers decreased by $26 \%$ from 26,429 in 2013 to 19,453 in 2019, with an average decrease of $-1,153 \pm 226 \mathrm{UDS} /$ year $(p=0.004)$ (Fig. 1).

In the urological departments, both the number of urodynamics performed $(-45 \% ; 11,758$ to 6,$409 ;-830 \pm$ $101 \mathrm{UDS} /$ year; $p<0.001)$ and the number of departments performing them ( $-20 \%$; 328 to 263 clinics; $-9 \pm 2$ clinics/ year; $p<0.001)$ decreased. Gynaecological departments also showed a decrease in the number of examinations $(-53 \% ; 1,861$ to $866 ;-170 \pm 9$ UDS/year; $p<0.001)$ and the number of performing departments $(-57 \% ; 159$ to 68 ; $-16 \pm 1$ clinics/year; $p<0.001)$. In paediatrics, on the other hand, there was an increase in the number of UDS (+40\%; 1,564 to 2,$192 ;+131 \pm 38$ UDS/year; $p=0.02)$ with a slight decrease in the number of departments $(-10 \% ; 92$ to $83 ;-2 \pm 1$ clinics/year; $p=0.02$ ). Multidisciplinary centres showed a non-significant decrease $(-12 \% ; 7,061$ to 6,$225 ;-186 \pm 146 \mathrm{UDS} /$ year; $p=0.3$ ) from 2013 to 2019 .
From 2018 to 2019, there seemed to be a drop from 7,347 to 6,226 . There was no significant change in the number of performing multidisciplinary departments (2013: 15 departments; 2019: 14 departments) (Fig. 2).

When analysing hospital characteristics, university hospitals showed a smaller decrease $(-11 \% ; 3,007$ to $2,685 ;-74 \pm 21 \mathrm{UDS} /$ year; $p=0.02)$ than non-university hospitals $(-28 \% ; 23,422$ to 16,$768 ;-1,079 \pm 225$ UDS/ year; $p=0.005)$. There was no significant change in the number of performing university hospitals (2013: 34 departments; 2019: 32 departments). Urological departments in a university hospital showed a smaller decrease than non-university urological departments $(-23 \%$ vs. $-50 \%)$. There were 54 centres in Germany with a continuous certification from 2013 to 2018. Certified pelvic floor centres showed a decrease of $-22 \%$ in UDS utilization $(5,137$ to 4,$009 ;-243 \pm 36$ UDS/year; $p<0.001)$.

Table 1 shows the age- and gender-dependent trends of UDS from 2013 to 2019 . There was no change in the sex ratio of patients undergoing UDS in hospitals from 2013 to 2019 (61\% male and 39\% female). Constant numbers were seen for children ( $\leq 19$ years: $-9 \% ; 1,371$ to $1,252 ;-28 \pm 16 \mathrm{UDS} /$ year; $p=0.2)$. The strongest decrease was seen in 60 - to 79 -year-olds $(-43 \% ; 9,792$ to $5,564 ;-736 \pm 50$ UDS/year; $p<0.001)$. The age group $20-39$ years also showed a decrease of $-21 \%(1,977$ to $1,561 ;-96 \pm 21 \mathrm{UDS} /$ year; $p=0.006), 40-59$ years $-33 \%$ $(5,825$ to 3,$925 ;-363 \pm 41$ UDS/year; $p<0.001), 60-79$ years $-43 \%(9,792$ to 5,$564 ;-736 \pm 50$ UDS/year; $p<$ $0.001)$, and $\geq 80$ years $-35 \%$ ( 1,886 to 1,$230 ;-97 \pm 14$ UDS/year; $p=0.001$ ) (Fig. 3). 
Table 1. Age- and gender-dependent development of UDS utilization from 2013 to 2019

\begin{tabular}{llll}
\hline Age group, years & All, $\%$ & Female, $\%$ & Male, $\%$ \\
\hline$\leq 19$ & $-9(-28 \pm 16$ UDS/year; $p=0.2)$ & $-5(-10 \pm 9$ UDS/year; $p=0.3)$ & $-12(-18 \pm 10$ UDS/year; $p=0.1)$ \\
$20-39$ & $-21(-96 \pm 21$ UDS/year; $p=0.006)$ & $-24(-48 \pm 10$ UDS/year; $p=0.007)$ & $-19(-53 \pm 17$ UDS/year; $p=0.03)$ \\
$40-59$ & $-33(-364 \pm 41$ UDS/year; $p<0.001)$ & $-39(-177 \pm 17$ UDS/year; $p<0.001)$ & $-27(-188 \pm 34$ UDS/year; $p=0.003)$ \\
$60-79$ & $-43(-736 \pm 50$ UDS/year; $p<0.001)$ & $-44(-290 \pm 20$ UDS/year $p<0.001)$ & $-43(-446 \pm 54$ UDS/year; $p<0.001)$ \\
$\geq 80$ & $-35(-97 \pm 14$ UDS/year; $p=0.001)$ & $-32(-32 \pm 7$ UDS/year; $p=0.005)$ & $-37(-65 \pm 11$ UDS/year; $p=0.002)$ \\
\hline
\end{tabular}

The $p$ values describe the linear regression from 2013 to 2019.

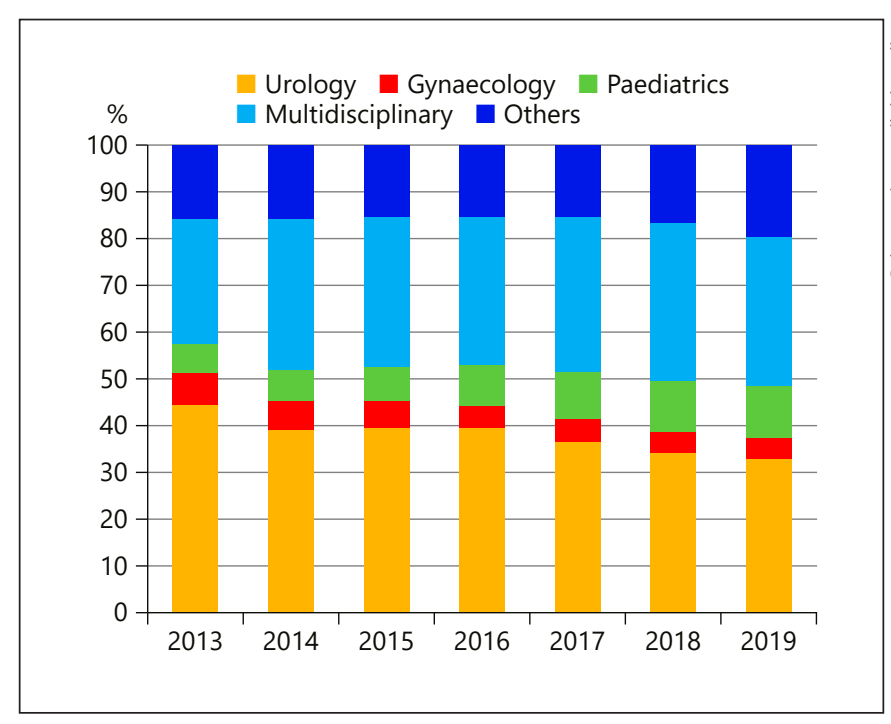

Fig. 3. Percentage distribution of UDS utilization in hospitals by department from 2013 to 2019.

\section{Discussion}

There was an overall decrease of $14 \%$ in UDS utilization in Germany from 2013 to 2018 (60,980 to 52,319). In the outpatient sector, there was a slight non-continuous decrease of $-11 \%$ from 34,551 to 30,652 from 2013 to $2018(p=0.06)$. In German hospitals, the UDS numbers decreased by $-26 \%$ from 26,429 in 2013 to 19,453 in 2019. In urological and gynaecological departments, both the number of UDS performed (urology: 11,758 to 6,409; $p<$ 0.001 ; gynaecology: 1,861 to $866 ; p<0.001)$ and the number of departments performing them (urology: 328 to 263 clinics; $p<0.001$; gynaecology: 159 to 68 ; $p<0.001)$ decreased markedly.

There are no other studies that show UDS utilization in recent years. Five studies showed trends in the period from 2000 to 2015. A US administrative claims database analysis showed an increase in UDS in men from 2000 to
2010 and a decrease from 2010 to 2012 [13]. A similar study analysed UDS in US women, with an increase from 2000 to 2009 and a decrease thereafter until 2012 [14]. Another study analysed a 5\% sample of US Medicare utilization records for UDS in women. It showed an increased use of UDS from 2000 to 2010. The strongest increase of $144 \%$ were among gynaecologists. UDS performed by urologists decreased by $3 \%$ [15]. A further US study of a Californian female population showed a decline in UDS utilization from 2010 to 2014, inconsistent with the results of the ValUE trial [16]. A Korean study also showed a decrease in UDS in the entire population from 2010 to 2015 using National Health Insurance data [17]. Our study can confirm the decreasing trend in the period from 2013 to 2015 mentioned by the Californian and Korean study. It furthermore shows a continuous decrease until 2019.

The evaluation of the total UDS numbers in German hospitals shows stable numbers from 2013 to 2016. Only from 2016 onwards does a decline become apparent. Nevertheless, it is important to analyse subgroups for a differentiated evaluation. Analysing departments, we found continuous decreases in the 2 specialities performing incontinence surgery. Gynaecological departments were the most affected $(-53 \% ;-169 \pm 9$ UDS/year; $p<$ 0.001). Urological departments are also seeing a sharp drop ( $-45 \% ; 830 \pm 101 \mathrm{UDS} /$ year; $p<0.001)$. On the one hand, we expected an influence of studies questioning the routine use of UDS in the diagnostics of uncomplicated SUI in women and LUTS in men $[7,8,10]$. This assumption is supported by the analysis of the age and gender distribution. In women, we see the strongest decrease in the age groups of $40-59$ and $60-79$ years $(-39 \%$ and $-43 \%)$, corresponding to the highest prevalence of female SUI $[18,19]$. In men, we see the strongest decrease in age groups $60-79$ and $\geq 80$ years, corresponding to the highest prevalence of SUI and LUTS in men [18]. Based on the decrease in UDS in the 2 surgical disciplines urol- 
ogy and gynaecology, and especially in both patient cohorts (male LUTS surgery and incontinence surgery), we suspect that UDS have become less important in the indication for surgery. Of course, there is a whole range of non-surgical indications for UDS. If we look at the UDS use of multidisciplinary departments, which will use UDS for non-surgical indications, we do not see a relevant decrease $(p=0.3)$. On the other hand, there also seems to be shifting effects among specialities, especially regarding urological departments. Paediatrics was the only department showing a significant increase in the number of UDS (+40\%; 1,564 to 2,$192 ;+131 \pm 38$ UDS/ year; $p=0.02$ ). However, age analysis showed constant numbers in children ( $-9 \% ; 1,371$ to 1,$252 ;-28 \pm 16$ UDS/ year; $p=0.2$ ). In recent years, there have been standardizations regarding UDS in children, but no major studies have changed its indications $[20,21]$. We hypothesize that there has been a shift in UDS in children from urology to paediatrics.

In contrast to the overall trend, no development is apparent in specialized multidisciplinary departments with relatively constant case numbers from 2013 to 2018 . There seems to be a drop in UDS in 2019, but an analysis of future years is necessary to show whether this is a random fluctuation or a real trend. These multidisciplinary departments are mainly centres for patients with spinal cord injuries or specialized centres for neurourology. The main reason for the constant numbers is that UDS are a recommended part of the standard diagnosis among neurourological patients [22]. Repeated UDS are even recommended among neurourological patients for clinical decision-making [23]. It can be speculated that an increasing number of patients with neurourological voiding dysfunctions are more often treated in specialized multidisciplinary departments than in urological departments.

In contrast to the overall trend, university hospitals showed a slight decrease (11\%) of UDS from 2013 to 2019. Focusing on urological departments, there was only a decrease of $23 \%$ in university hospitals in contrast to $50 \%$ in non-university hospitals. A Dutch survey amongst urologists and gynaecologists after the ValUE and VUSIS trials showed that physicians in academic hospitals were more likely to use UDS in routine diagnostics for SUI in women. The reasons given were the additional value, especially the pressure transmission ratio and the presence of detrusor overactivity [24]. Another influencing factor may be the public ownership of university hospitals with higher subsidies for investment costs by federal states [25].
High costs based on time, personnel, and material expenditure are often cited as a reason for the decline of UDS [6]. A secondary analysis from the ValUE study calculated savings of USD 13-33 million in avoiding UDS in uncomplicated SUI patients [6]. A British study calculated savings in favour of UDS of GBP 138 (159 EUR) per case in women with SUI or stress-predominant mixed urinary incontinence because of a reduction in surgery numbers [26]. The decisive factor here is the level of remuneration. In Germany in 2021, an outpatient UDS is remunerated at $95.11 \mathrm{EUR}$, which is quite small considering the resources required [27]. In view of this, it is surprising that there is a smaller decline in UDS in the outpatient sector $(-11 \% ;-1,185 \pm 445$ UDS/year; $p=0.06)$ than in hospitals because doctors generally have fewer personnel and financial resources than hospitals. We hypothesize that there may also be shifting effects from hospitals to the outpatient sector. A relevant factor may be the increase in outpatient medical care centres owned by hospitals. Hospitals transfer outpatient treatment from their hospital outpatient clinic to their external medical care centres. From 2013 to 2019, there was an overall increase of $100 \%$ in outpatient medical care from 795 to 1,589 centres in Germany [28].

The use of quality reports enables a very accurate representation of the health care situation in hospitals. Additionally, patient data of the InGef showed good overall concordance with the German population [12]. However, there are several limitations. The case numbers in the quality reports are slightly higher than the reality, as case numbers from 1 to 3 are coded as 3 . The quality reports are prepared by hospitals and may be subject to documentation errors. Age and gender include only inpatient cases. Therefore, there may be a bias for patients with more complex diseases or higher comorbidities. In the InGef database, it is not possible to discriminate medical specializations. In both claims databases, the clinical data are missing. Therefore, we cannot characterize the indication and quality of UDS. It is possible to make rough assumptions regarding the indication depending on the performing department and the age of patients. This is the first study showing recent trends in UDS utilization in a European country and different developments depending on the clinical specialities. We assume a decreasing significance for UDS in the indication for surgery. Nevertheless, we can only speculate about the reasons for these trends. Additional studies have to investigate the reasons for the reduced numbers by surveying physicians performing UDS. 


\section{Acknowledgments}

This project was developed within the framework of the working group "Health Services Research, Quality and Economics" of the DGU e.V. We thank Isabelle Braun (Deutsche Kontinenz Gesellschaft e.V.) for supporting data retrieval.

\section{Statement of Ethics}

The study is exempt from ethics committee approval due to the nature of data (health insurance data and public accessible hospital quality reports). Also, written informed consent was not needed.

\section{Conflict of Interest Statement}

Johannes Huber and Martin Baunacke received non-financial support from Coloplast $\mathrm{GmbH}$ during the conduct of the study. Marcel Schmidt is an employee of Coloplast GmbH. Nicole Eisenmenger is the Founder and Managing Director of RI Innovation $\mathrm{GmbH}$. All other authors have no conflicts of interest to declare.

\section{Funding Sources}

Coloplast $\mathrm{GmbH}$ initiated and paid for the data retrieval by using the InGef research database and provided the results free of charge as part of a scientific cooperation.

\section{Author Contributions}

Martin Baunacke and Johannes Huber contributed to protocol/project development. Marcel Schmidt, Nicole Eisenmenger, and Albert Kaufmann contributed to data collection or management. Martin Baunacke and Isabel Leuchtweis contributed to data analysis. Martin Baunacke, Johannes Huber, Christian Thomas, Isabel Leuchtweis, Christer Groeben, and Angelika Borkowetz contributed to manuscript writing/editing.

\section{Data Availability Statement}

German hospital quality reports are publicly accessible. Health insurance data are accessible by using the InGef (Institute for Applied Health Research) Research Database.

\section{References}

1 Schafer W, Abrams P, Liao L, Mattiasson A, Pesce F, Spangberg A, et al. Good urodynamic practices: uroflowmetry, filling cystometry, and pressure-flow studies. Neurourol Urodyn. 2002;21(3):261-74.

2 Collins CW, Winters JC, American Urological A, Society of Urodynamics Female Pelvic M, Urogenital R. AUA/SUFU adult urodynamics guideline: a clinical review. Urol Clin North Amvii. 2014;41(3):353-62.

3 Gammie A, Clarkson B, Constantinou C, Damaser M, Drinnan M, Geleijnse G, et al. International Continence Society guidelines on urodynamic equipment performance. Neurourol Urodyn. 2014;33(4):370-9.

4 Gammie A, Almeida F, Drake M, Finazzi Agro E, Kirschner-Hermanns R, Lemos N, et al. Is the value of urodynamics undermined by poor technique?: ICI-RS 2018. Neurourol Urodyn. 2019;38(Suppl 5):S35-9.

5 Nobrega MM, Auge AP, de Toledo LG, da Silva Carramao S, Frade AB, Salles MJ. Bacteriuria and urinary tract infection after female urodynamic studies: risk factors and microbiological analysis. Am J Infect Control. 2015; 43(10):1035-9.

6 Norton PA, Nager CW, Brubaker L, Lemack GE, Sirls LT, Holley R, et al. The cost of preoperative urodynamics: a secondary analysis of the ValUE trial. Neurourol Urodyn. 2016; 35(1):81-4.

7 Nager CW, Brubaker L, Litman HJ, Zyczynski HM, Varner RE, Amundsen C, et al. A randomized trial of urodynamic testing before stress-incontinence surgery. N Engl J Med. 2012;366(21):1987-97.
8 van Leijsen SAL, Kluivers KB, Mol BWJ, Hout J, Milani AL, Roovers JWR, et al. Value of urodynamics before stress urinary incontinence surgery: a randomized controlled trial. Obstet Gynecol. 2013;121(5):999-1008.

9 EAU guidelines. In: Edn. presented at the EAU Annual Congress Milan 2013. Arnhem, The Netherlands: EAU Guidelines Office; 2013.

10 Clement KD, Burden H, Warren K, Lapitan MC, Omar MI, Drake MJ. Invasive urodynamic studies for the management of lower urinary tract symptoms (LUTS) in men with voiding dysfunction. Cochrane Database Syst Rev. 2015;(4):CD011179.

11 Lewis AL, Young GJ, Selman LE, Rice C, Clement C, Ochieng CA, et al. Urodynamics tests for the diagnosis and management of bladder outlet obstruction in men: the UPSTREAM non-inferiority RCT. Health Technol Assess. 2020;24(42):1-122.

12 Andersohn F, Walker J. Characteristics and external validity of the German Health Risk Institute (HRI) database. Pharmacoepidemiol Drug Saf. 2016;25(1):106-9.

13 Conover MM, Jonsson Funk M, Kinlaw AC, Borawski KM, Wu JM. Trends and patterns of urodynamic studies in U.S. males, 2000-2012. PLoS One. 2015;10(7):e0133657.

14 Conover MM, Jonsson Funk M, Kinlaw AC, Connolly A, Wu JM. Trends and patterns of urodynamic studies in U.S. women, 20002012. Obstet Gynecol. 2015;125(3):559-65.
15 Reynolds WS, Ni S, Kaufman MR, Penson DF, Dmochowski RR. Trends in urodynamics in U.S. female medicare beneficiaries, 2000 2010. Neurourol Urodyn. 2015;34(5):420-3.

16 Lippmann QK, Diwadkar GB, Zhou H, Menefee SA. Trends in urodynamics study utilization in a Southern California managed care population. Am J Obstet Gynecol. 2015; 213(5):724-6.

17 Baek MJ, Park S, Kim KH, Kim YH, Kim WK, Sun HY, et al. National trend of uroflowmetry, urodynamic study and cystoscopy considering the change in the population structure in Korea from 2010 to 2015. J Korean Med Sci. 2018;33(20):e145.

18 Irwin DE, Milsom I, Hunskaar S, Reilly K, Kopp Z, Herschorn S, et al. Population-based survey of urinary incontinence, overactive bladder, and other lower urinary tract symptoms in five countries: results of the EPIC study. Eur Urol. 2006;50(6):1306-14; discussion 14-5.

19 Milsom I, Gyhagen M. The prevalence of urinary incontinence. Climacteric. 2019;22(3): $217-22$.

20 Samijn B, Van Laecke E, Vande Walle J, Pascal A, Deschepper E, Renson C, et al. Uroflow measurement combined with electromyography testing of the pelvic floor in healthy children. Neurourol Urodyn. 2019;38(1):231-8.

21 Bauer SB, Nijman RJ, Drzewiecki BA, Sillen U, Hoebeke P; International Children's Continence Society Standardization Subcommittee. International Children's Continence Society standardization report on urodynamic studies of the lower urinary tract in children. Neurourol Urodyn. 2015;34(7):640-7. 
22 EAU guidelines. In: Edn. presented at the EAU Annual Congress Amsterdam 2020. Arnhem, The Netherlands: EAU Guidelines Office; 2020.

23 Bellucci CH, Wollner J, Gregorini F, Birnbock D, Kozomara M, Mehnert U, et al. Neurogenic lower urinary tract dysfunction--do we need same session repeat urodynamic investigations? J Urol. 2012;187(4):1318-23.
24 Mengerink BB, Nelen WLDM, van Leijsen SAL, Heesakkers JPFA, Kluivers KB. De-implementation of urodynamics in The Netherlands after the VALUE/VUSIS-2 results: a nationwide survey. Int Urogynecol J. 2018; 29(9):1261-77.

25 Pilny A. Explaining differentials in subsidy levels among hospital ownership types in Germany. Health Econ. 2017;26(5):566-81.

26 Homer T, Shen J, Vale L, McColl E, Tincello DG, Hilton P, et al. Invasive urodynamic testing prior to surgical treatment for stress urinary incontinence in women: cost-effectiveness and value of information analyses in the context of a mixed methods feasibility study. Pilot Feasibility Stud. 2018;4:67.
27 Kassenärztliche_Bundesvereinigung. Online-Version des EBM. Available from: https: //www.kbv.de/html/online-ebm.php.

28 Kassenärztliche_Bundesvereinigung. Gesundheitsdaten: Medizinische Versorgung. Available from: https://gesundheitsdaten. kbv.de/cms/html/17021.php. 